Original Research Paper Medical Science

\title{
A CASE PRESENTATION: RADIOLOGICAL AND PATHOLOGICAL ROLE IN DIAGNOSIS OF BENING RENAL MASS - ONCOCYTOMA
}

\section{B. keerthana}

\begin{tabular}{ll}
\hline N. Siva Durgesh ${ }^{*}$ & ${ }^{*}$ Corresponding Author \\
\hline B. Anji Naik & \\
\hline
\end{tabular}

ABSTRACT Oncocytoma is benign renal tumor. Incidence of oncocytoma is $3 \%-7 \%$ among all the kidney tumors. A 45 year old female was presented with complaints of pain in right loin since 1 year. Conclusion Renal oncocytoma is difficult diagnosis to conclude with only radiological features and pathological findings, the diagnosis can be confirmed only by chemical staining in histo pathological examination, as radiologically oncocytoma closely resembles RCC and is challenging to differentiate between the two.

KEYWORDS : Radiological, Pathological benign, Renal mass oncocytoma.

\section{INTRODUCTION}

Oncocytoma is benign renal tumor. Incidence of oncocytoma is $3 \%-7 \%$ among all the kidney tumors. An oncocytoma is a tumor made up of oncocytes, epithelial cells characterized by an excessive amount of mitochondria resulting in an abundant acidophilic, granular cytoplasm, derived from distal renal tubules.

\section{Case Presentation}

A 45 year old female was presented with complaints of pain in right loin since 1 year. Insidious onset, progressive in nature, dragging type of pain, no aggravating factors, relieved on medication. History of hematuria since 1 month, loss of appetite. No history of vomiting, trauma, fever.

On clinical examination $-6^{*} 7 \mathrm{~cm}$ mass palpable in right loin. No tenderness present, no warmth, firm in consistency, mass is bimanually palpable and ballotable, no renal angle tenderness, For which ultrasound abdomen and pelvis was done, which revealed a $7.8 \mathrm{~cm} * 5.7 \mathrm{~cm}$ renal mass with possible malignancy ( $R C C$ ) , no evidence of renal vein or IVC thrombosis.

Further CECT abdomen was done which showed large heterogeneously enhancing exophytic mass lesion of size $7.2 * 6.4 \mathrm{~cm}$ involving anterior cortex of upper and middle pole of right kidney. Central scar noted, no evidence of calcifications. washout was noted in venous and delayed phase. Possibility of oncocytoma.

Radiological Findings: They appear as sharply demarcated lesions of variable size, but often large at presentation. The characteristic sign is presence of central stellate scar but is seen only in $1 / 3^{\text {rd }}$ of patients. Central scar is also demonstrated in cases of RCC (Fig l).

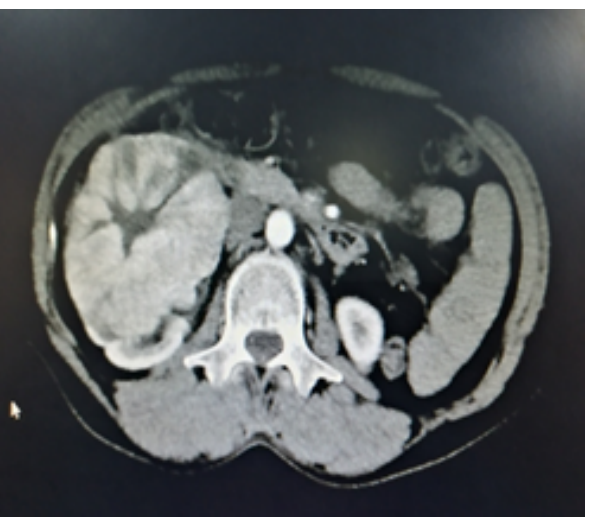

The patient was planned for right radical nephrectomy under GA. Intra-operatively - right kidney along with mass of size $7^{\star} 6 \mathrm{~cm}$ was excised along with gerotas fascia, right adrenal gland was preserved. Specimen was sent for HPE. Postoperative period was uneventful and wound was healthy, DT removal done on pod 2 and pod 8 suture removal done. HPE report confirmed the diagnosis of oncocytoma. Immunohistochemistry further confirmed the diagnosis of oncoytoma.

Gross Features: Well circumscribed, encapsulated, solid , homogenous, mahogany or brownish yellow , stellate central scar in larger lesions. (Fig-2)



Fig-2

Central stellate scar

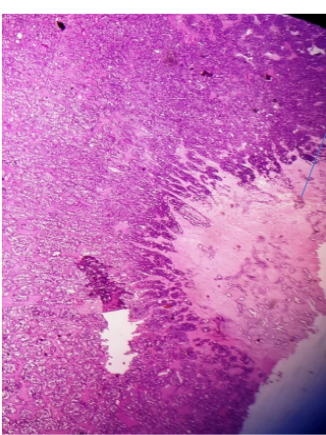

Fig 3

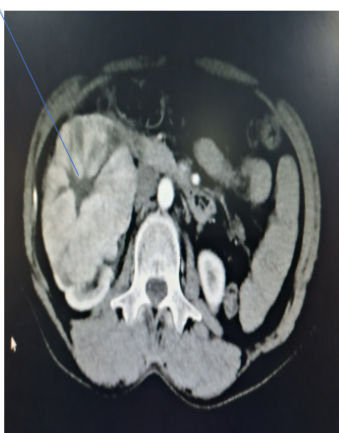

Fig 4
Pathological Findings It is benign tumor of uniform round / polygonal cells with abundant, intensely eosinophilic and granular cytoplasm with uniform small, round and central nuclei with evenly dispersed chromatin. Histologically, the 
greratest dilemma arises from distinguishing chromophobe and clear cell RCC from oncocytoma.



Fig-5: H\&e 40x: The Lesion Shows Oval To Polyhedral Cells With Vesicular Nuclei, Dispersed Chromatin And Abundant Granular Eosinophilic Cytoplasm Within Intervening Hyalinized Stroma.

Immunohistochemistry Hale colloidal staining is classic differentiating marker for oncocytoma. Cytokeratin profiles are helpful in distinguishing these histologic findings. expression of CK - 7 is seen in $66 \%$ of chromophobe $\mathrm{RCC}$ and only $5 \%$ of oncocytomas. Other markers useful for differentiating RCC from oncocytoma include - pax-2, CK20, Cd1 5 claudin 7 and 8, c-kit expression, s-100, NPM and LMP2.
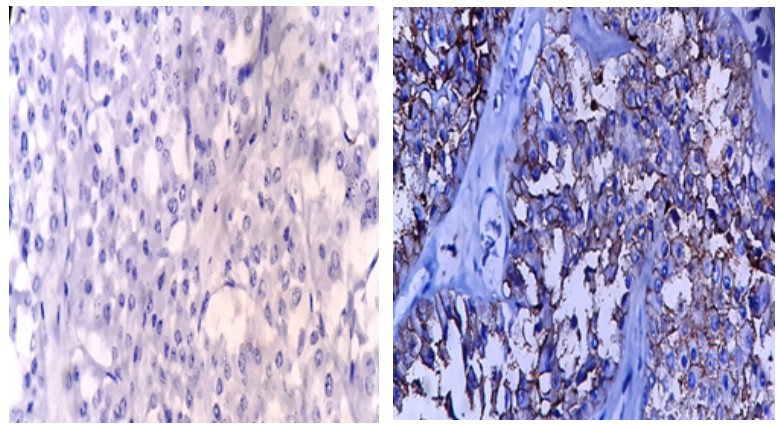

Fig 6- Ck7 Negative, Fig-7 Cd15 - Shows Moderate Membranous Positivity In Lesional Cells

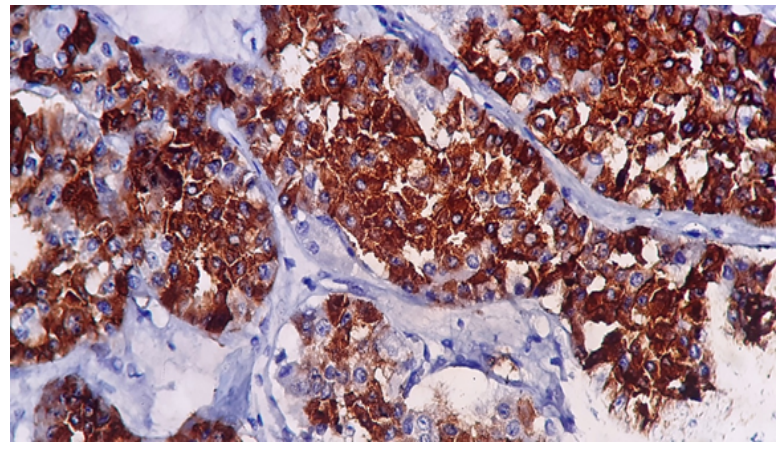

Fig 8: Ck20 -intense Cytoplasmic Positivity In Lesional Cells

This case revealed -

1. CK7 - majority of lesional cells negative, scattered cells positive.
2. CK20-diffusely positive in lesional cells

3. CD15 - very patchy strong cytoplasmic positivity in lesional cells.

Therefore further diagnosis of oncocytoma is confirmed.

\section{DISCUSSION}

Oncocytoma are common in older patients, and are more common in males than females. The most common genetic abnormality is loss of heterozygosity at chromosomes lor 14 . Other common cytogenetic findings include loss of $\mathrm{Y}$ chromosome and chromosome 14q and rearrangements of llql3.

Oncocytomas are genotypically distinct from RCC. Chromophobe RCC particulary eosinophilic variant closely resemble oncocytoma histologically. The greatest clinical dilemma remains the inability to confidently differentiate between renal oncytoma and $\mathrm{RCC}$ on clinical or radiographic testing.

Radigraphically the typical stellate scar gives a clue to diagnosis of oncocytoma. In this case, pathologically resembled papillary type $\mathrm{RCC}$, due to papillary projections and some amount of necrosis. Therefore for further evaluation and confirmation the slides were sent to path care for chemical staining. There are 3 cellular patterns of oncocytoma including organoid, tubulocystic and mixed pattern. The organoid pattern is characterised by nests of tumour cells surrounded by a reticular framework of thin blood vessels and stroma. Abundant vascularity in the organoid pattern may account for the arterial enhancement of oncocytomas. In the organoid pattern, tightly packed tumour nests may explain washout patterns in the venous phase owing to its high cellularity. $5 \%$ are bilateral or multifocal, may invade capsule or renal vein. $20 \%$ have gross hemorrhage but necrosis is rare. Frozen section analysis at time of surgery may not realiably distinguish oncocytoma from RCC.

CONCLUSION: Renal oncocytoma is difficult diagnosis to conclude with only radiological features and pathological findings, the diagnosis can be confirmed only by chemical staining in histo pathological examination, as radiologically oncocytoma closely resembles RCC and is challenging to differentiate between the two. 